OPEN

SUBJECT AREAS:

PHYSICS

NANOSCIENCE AND

TECHNOLOGY

ENGINEERING

Received

24 January 2014

Accepted

21 August 2014

Published

6 October 2014

Correspondence and requests for materials should be addressed to J.Z. (junyanzh@yahoo. com) or G.S. (gsu@ ucas.ac.cn)

\section{From sphere to polyhedron: A hypothesis on the formation of high-index surfaces in} nanocrystals

\author{
Yan Zhou', Junyan Zhang', Gang Su' \& Jiangong Li ${ }^{3}$
}

\begin{abstract}
'State Key Laboratory of Solid Lubrication, Lanzhou Institute of Chemical Physics, Chinese Academy of Sciences, Lanzhou 730000, China, ${ }^{2}$ School of Physics, University of Chinese Academy of Sciences, Beijing 100049; China, ${ }^{3}$ Institute of Materials Science and Engineering, Lanzhou University, Lanzhou 730000, China.
\end{abstract}

The morphology of tetrahexahedral nanocrystals could be understood on the basis of a hypothesis that the atoms or molecules on or near spherical surfaces can migrate till reaching their equilibrium positions. Such migration of atoms/molecules is shown to be closely related to the formation of high-index surfaces in nanopolyhedrons. On account of this hypothesis, a theoretical calculation about the indices of the surfaces in tetrahexahedrons is found in good agreement with the empirical results. A group of high-index surfaces for nanocrystals that can be formed under certain environments are thus predicted. This study may provide a novel idea for preparing the catalysts at nanoscale.

ecently, polyhedral nanocrystals of noble-metals, such as $\mathrm{Au}, \mathrm{Pd}, \mathrm{Pt}$, etc. $^{1-13}$, have attracted much attention for their notable catalytic activities ${ }^{1-5}$. Among these nanocrystals, tetrahexahedron becomes a hot issue for its high-index surfaces and unknown formation mechanism ${ }^{3,7-12}$. This work focuses on the unusual morphology of tetrahexahedrons, and a hypothesis to understand the formation mechanism of the high-index surfaces in tetrahexahedrons is proposed.

In earlier works, the surfaces in convex tetrahexahedrons are discovered to be $\{210\},\{520\},\{730\}$ and so on ${ }^{10}$. These surfaces have general characters: (i) they are mainly composed of (210) steps and (310) steps; (ii) these two kinds of steps present erratically; and (iii) the integration of these randomly-ordered steps results in constant angles between any two adjacent surfaces. Currently, no well-accepted mechanism explains the formation of such a high-index surface with disordered steps but constant angles. This puzzling character accounts for different recognitions of the high index: the integration of repeated (210) steps leads to the index of $\{210\}$, the integration of two equal parts of (210) steps and (310) steps leads to the index of $\{520\}$, and the integration of three equal parts of (210) steps, (210) steps and (310) steps leads to $\{730\}$ (Fig. 1). Each of these indices can be read from a certain segment in a tetrahexahedral surface (see HRTEM images of Fig. 3a in Ref. 8 and Fig. 2E in Ref. 10), but it is difficult to assure the nature that the structures are covered randomly by (210) steps and (310) steps, and in particular, many are not in complete agreement with the measured values (Fig. 1).

There are recent works showing that it is a transcendental approach to take a nano or micro particle as a holistic one $^{14}$. Here we attempt to take the single crystal polyhedrons of noble-metals as cooperative structures, which behave like gigantic molecules rather than reductionistic ones. This assumption could lead to an explanation about the formation of the high-index surfaces in the convex tetrahexahedron. The same analysis is also applied to discuss the formation of the high indices in concave tetrahexahedrons.

\section{Results}

Since the high-index surfaces in noble-metal tetrahexahedrons are thermally stable and constant ${ }^{10}$, they should be formed determinately by certain driving forces. To explore these driving factors, the formation processes of these tetrahexahedral nanocrystals are summarized here: (i) they are all single nanocrystals with face-centered cubic structures $(f c c)$; (ii) the amorphous or single crystal spheres present as precursors of the tetrahexahedrons ${ }^{10}$; and (iii) the tetrahexahedrons are formed via the exterior atoms/molecules migrating under certain conditions.

These general characters give clues that can connect the tetrahexahedrons of cubic structures to the spheres of amorphous or single crystal structures. During the transition from an amorphous sphere to a crystal polyhedron, two ways may lead the atoms/molecules to the equilibrium knots: one is that the interior atoms/molecules crystallize in-situ, the other is that the exterior atoms/molecules migrate to the crystallographically favorite sites. 


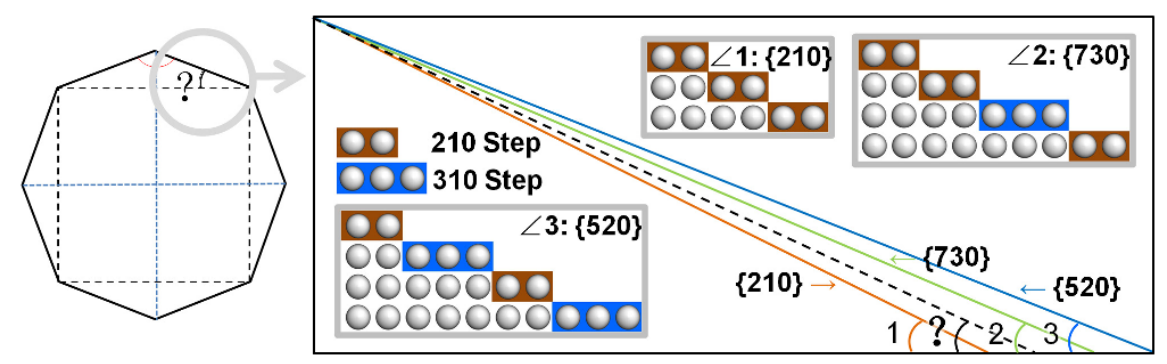

Figure 1 Various indices of the surface of a tetrahexahedron. Different approaches result in different indices: repeated (210) steps lead to the index of $\{210\}\left(\angle 1=26.57^{\circ}\right)$, two $(210)$ steps and one (310) step lead to $\{730\}\left(\angle 2=23.20^{\circ}\right)$, and equal amounts of $(210)$ steps and (310) steps lead to the index of $\{520\}\left(\angle 3=21.80^{\circ}\right)$. All these indices differ from the empirical results $(\angle$ ? $)$.

For the latter, a crystal sphere should be an intermediate before the exterior atoms/molecules migrate to the equilibrium sites. Assuming that the volume is constant during the transition from a crystal sphere to a crystal polyhedron, the emigrating atoms/molecules and the immigrating atoms/molecules should have equal volumes. This assumption highlights the migrating of the exterior atoms/ molecules when a sphere transforms into a polyhedron. In a given two-dimensional (2D) crystal disk of square cell structure, the exterior atoms/molecules will move till a crystal polygon forms (Fig. 2). The net effect, at the end, is that some atoms/molecules in certain zones emigrate while some others immigrate, and the surfaces play roles as the migrating channels.

Considering that both the sphere and the polyhedron act at nanoscale, the local environment can be taken to be homogeneous one, under which the sphere and the polyhedron tend to be as symmetrical as possible for the extrinsic factors seldom break their intrinsic symmetries ${ }^{15}$. The following restrictions towards understanding the transformation from an amorphous sphere to a crystal polyhedron with cubic structure should be taken into account: (i) the amorphous precursor is a regular sphere with rotational symmetry ( $K_{h}$ group); (ii) the intermediate of the crystal sphere and the crystal polyhedron are of cubic/octahedral symmetry ( $O_{h}$ group); (iii) the crystal polyhedron is covered by identical surfaces of $\{h k l\}$, which assures the symmetry of the crystal particle and the equal energy all over the surfaces; (iv) the volume remains constant during the transformation; and (v) the energy aroused by those migrating atoms/molecules is as low as possible. Now, we may assume that the energy to migrate atoms/molecules per unit volume is $E_{m}$, and the energy to expose a new surface per unit area is $E_{s}$. It is clear that $E_{m}$ is mainly related to the kinetic energy of the migrating atoms/molecules, and $E_{s}$ is primarily a thermodynamic parameter determined by the crystal index $\{h k l\}$. Then, the reshaping energy to migrate all involved atoms/ molecules via an equilibrium or quasi-equilibrium process at a given condition would be

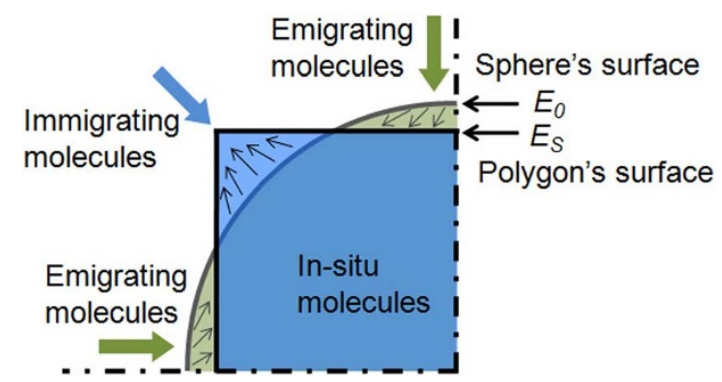

Figure 2 2D structures: a sphere transforms into a crystal polyhedron with square cell structure via exterior atoms/molecules migrating across the surfaces. The sphere is colored by green and the crystal polyhedron is blue. $E_{0}$ is the surface energy of the initial sphere, and $E_{s}$ is the surface energy of the polygon.

$$
E=\int_{-\Delta V}^{\Delta V} E_{m} \mathrm{~d} V+\int_{0}^{S} E_{S} \mathrm{~d} S-E_{0}
$$

where $\Delta V$ is the change of the volume of the sphere, $S$ is the area of the surface of the polygon, and $E_{0}$ is a constant of the surface energy of the initial sphere.

It should be mentioned here that without etching or depositing, the energy aroused by the immigrating atoms/molecules $\left(E_{m} \cdot \Delta V\right)$ should offset the energy aroused by the emigrating atoms/molecules $\left(-E_{m} \cdot \Delta V\right)$ accurately or approximately, and the term $\int_{-\Delta V}^{\Delta V} E_{m} \mathrm{~d} V$ in Equation (1) is zero accurately or approximately, where "accurately" refers to the equilibrium process, while "approximately" to the quasiequilibrium process. By expressing the sum of $\int_{-\Delta V}^{\Delta V} E_{m} \mathrm{~d} V$ and $-E_{0}$ by a constant $a$, Equation (1) can be rewritten as

$$
E=\int_{0}^{S} E_{S} \mathrm{~d} S+a
$$

From Equation (2) it can be observed that the energy to reshape the particle mainly depends on the term $\int_{0}^{S} E_{S} \mathrm{~d} S$. The possible ways, via which an amorphous sphere can be reshaped into a crystal with specific symmetry, are listed in Table 1.

Table 1 shows that the term $\int_{0}^{S} E_{S} \mathrm{~d} S$ is determined by the product of $E_{h k l}$ and $S$ under a given condition. The value of $E_{h k l} \cdot S$ varies in different systems or conditions. If the process is mainly controlled by the surface energy, and a smaller surface area is allowed, the structure tends to be the morphology of a polyhedron closer to a regular sphere such as a complex convex tetrahexahedron; on the contrary, if a bigger surface area is allowed, it tends to be a simple polyhedron with low indices or even be a concave polyhedron. This uncertainty is consistent with the observation that the morphologies of single nano crystals with cubic structures are diverse $\mathrm{e}^{1-13,16-20}$.

Specifically, the last row in Table 1 presents the case that an amorphous sphere turns directly into a crystal sphere with all atoms/molecules crystallizing in-situ. The surface area of the crystal sphere has the minimum value of 1 (compared with the initial amorphous sphere), which may decrease the value of $\int_{0}^{S} E_{S} \mathrm{~d} S$. Such a crystal sphere is, however, unstable for it is covered by the surfaces with continuously changed indices and has an unequal surface energy everywhere. It accounts for the phenomenon that the crystal spheres are generally observed as unstable by-products with the polyhedrons ${ }^{8}$. For the crystal polyhedrons, including tetrahedrons, cubes and octahedrons, they are stable for being covered by facets with low indices and equal surface energy. Table 1 reveals that the 


\begin{tabular}{|c|c|c|c|c|c|c|c|}
\hline $\begin{array}{l}\text { Initial state } \\
\text { (Amorphous) }\end{array}$ & $\begin{array}{l}\text { Final state } \\
\text { (Crystal) }\end{array}$ & $\begin{array}{l}\text { Crystal } \\
\text { symmetry }\end{array}$ & $\begin{array}{l}\text { Surface } \\
\text { index }\end{array}$ & $\begin{array}{l}\text { Volume of the } \\
\text { migrating atoms }\end{array}$ & $\begin{array}{l}\text { Surface area } \\
\qquad\left(S / S_{0}\right)\end{array}$ & $\begin{array}{l}\text { Surface } \\
\text { energy }\end{array}$ & $\int_{0}^{S} E_{S} \mathrm{~d} S$ \\
\hline 0 & & $T_{d}$ & $+\left\{\begin{array}{lll}1 & 1 & 1\end{array}\right\}$ & 0.26 & 1.49 & Equal & $E_{111} \times 1.49 S_{0}$ \\
\hline $\begin{array}{l}\text { Surface area: } \\
\qquad S_{0}=1\end{array}$ & & $O_{h}$ & $\{100\}$ & 0.16 & 1.24 & Equal & $E_{100} \times 1.24 S_{0}$ \\
\hline $\begin{array}{l}\text { Volume: } \\
\qquad V_{0}=1\end{array}$ & & $\mathrm{O}_{\mathrm{h}}$ & $\left\{\begin{array}{lll}1 & 1 & 1\end{array}\right\}$ & 0.13 & 1.18 & Equal & $E_{111} \times 1.18 S_{0}$ \\
\hline $\begin{array}{l}\text { Symmetry: } \\
K_{h} \text { group; }\end{array}$ & & & $\begin{array}{c}\{h k 0\} \\
\{2.08,1,0\}\end{array}$ & $\begin{array}{c}0<\Delta V<0.13 \\
0.08\end{array}$ & $\begin{array}{c}0<S<1.18 \\
1.06\end{array}$ & Equal & $E_{2.08,1,0} \times 1.06 S_{0}$ \\
\hline $\begin{array}{l}\text { Surface energy: } \\
E_{0} \text { (Equal } \\
\text { everywhere) }\end{array}$ & 0 & $\mathrm{O}_{\mathrm{h}}$ & Various & 0 & 1.0 & $\begin{array}{l}\text { Unequal } \\
\text { everywhere }\end{array}$ & / \\
\hline
\end{tabular}

more complicated the polyhedron is, the smaller the surface area is, and this trend allows for the introduction of more complicated polyhedrons to get smaller values of $\left(E_{h k l} \cdot S\right)$.

The tetrahexahedron with $O_{h}$ symmetry and cubic structure is covered by 24 identical facets of $\{h k 0\}$. According to the above assumptions, the formation of these unusual facets can be proposed, as depicted in Fig. 3. It is seen that the initial state is a sphere under possible conditions (for example, the tetrahexahedral Pt nanocrystals are formed if Pt polycrystals are broken into smaller spheres by ultrasonic potential of square-wave $e^{8,10}$ ). When the subsequent reshaping process transforms the sphere into a polyhedron, the interior atoms/molecules reach the lattice knots earlier for in-situ crystallization, and the exterior atoms/molecules reach their positions later for migrating. These two different ways to reach the final positions divide the sphere into two suppositional parts: a stable core and a movable shell. After the core is crystallized, the shell keeps tuning itself till it meets the following requirements: (i) to be crystallographically congruous to the crystallized core; (ii) to be equal in surface energy; and (iii) to be symmetrical. The second and the third requirements imply that the division between the core and the shell is determined by geometrical effects besides dynamical effects. Concerning the determinate term $E_{h k l} \cdot S$, the dynamics affects $E_{h k l}$ and $S$ differently (note that the thermodynamics requires the surfaces with low indices, while the polyhedrons covered by low-index surfaces tend to be with larger surface areas, as shown in Table 1), while the geometry only affects $S$. When searching for the minimum value of $\left(E_{h k l} \cdot S\right)$, some geometrically critical points are discovered to balance exactly $E_{h k l}$ and $S$.

The side length of $\sqrt{2} r$ is one of the critical values in this transition. At this critical state, the core is a cube with side length of $\sqrt{2} r$, the exterior sphere is divided into 6 zones unconnected topologically (when the side length is bigger than $\sqrt{2} r$, the exterior zones will be divided into completely unconnected zones; on the other hand, when the side length is smaller than $\sqrt{2} r$, the above 6 zones will be connected into one zone; so here $\sqrt{2} r$ is a critical value of the side length), and both of these two parts are with symmetry of $O_{h}$ group. Each of the exterior zones attaching to a square plane of square symmetry $\left(D_{4}\right.$ group) will tune itself till it reaches the final state with $D_{4}$ symmetry and smaller surface areas. Thus, each zone reforms itself into a pyramid, and the particle ends with a tetrahexahedral nanocrystals (Fig. 3).

The parameters including $r, L, h$ and $\alpha$, as indicated in Fig. 3, are determined by the critical values: when $L$ reaches $\sqrt{2} r, h=\left(\frac{\pi}{3}-\frac{\sqrt{2}}{2}\right) r$, and $\operatorname{tg}\left(\frac{\alpha}{2}\right)=\frac{L}{2 h}=1:\left(\frac{\sqrt{2}}{3} \pi-1\right)=2.08$. Accordingly, the angles in a perfect tetrahexahedron are $\alpha=128.7^{\circ}$ and $\beta=141.3^{\circ}$. Fig. 4 a compares the calculations to the empirical results ${ }^{1,8}$. In investigating the determinate term $\left(E_{s} \cdot S\right)$ for the tetrahexahedron, the surface area is $1.06 S_{0}$ and the volume of the migrating atoms/molecules is $0.08 V_{0}$, which are relatively small, while $E_{h k l}$ is bigger than each value of the lowindex facets (Table 1). This suggests that the requirement of smaller surface areas $(S)$ is relatively prior to that of low surface energy $\left(E_{s}\right)$ here.

The hypothesis appears to give a possible explanation of the origin of the high indices in tetrahexahedrons of noble-metals, where an enveloping line $\left[y=\left(\frac{\sqrt{2}}{3} \pi-1\right) x+a\right]$ for various indices from various segments of the facets can be deduced. The side length of $2 r$ is another critical point besides $\sqrt{2} r$, and in this case, the sphere is transformed into a concave tetrahexahedon with parameters shown in Fig. $4 b^{1}$. Related works evidenced that these concave tetrahexahedrons are covered by high-index surfaces ${ }^{1,3,4,16-20}$. Our theoretical calculation gives that the index of the facets in the concave tetrahexahedron is $\{1,(1-\pi / 6), 0\}$, which predicts that the facets are approximate to the surfaces in the convex tetrahexahedron. Jin et al. observed that the surfaces of the concave tetrahexahedrons were composed of (310) steps and (210) steps, and the index was recognized as $\{730\}^{1}$. Although these two surfaces are coincident in their indices, their origins are different, because the index $\{730\}$ in convex tetrahexahe-

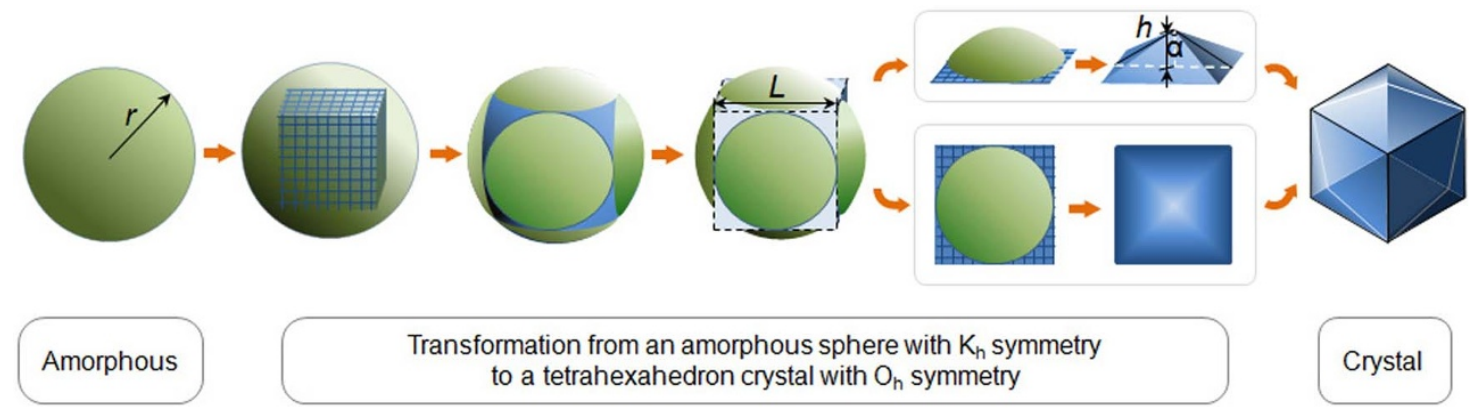

Figure 3 The formation process of a tetrahexahedral nanocrystal. 

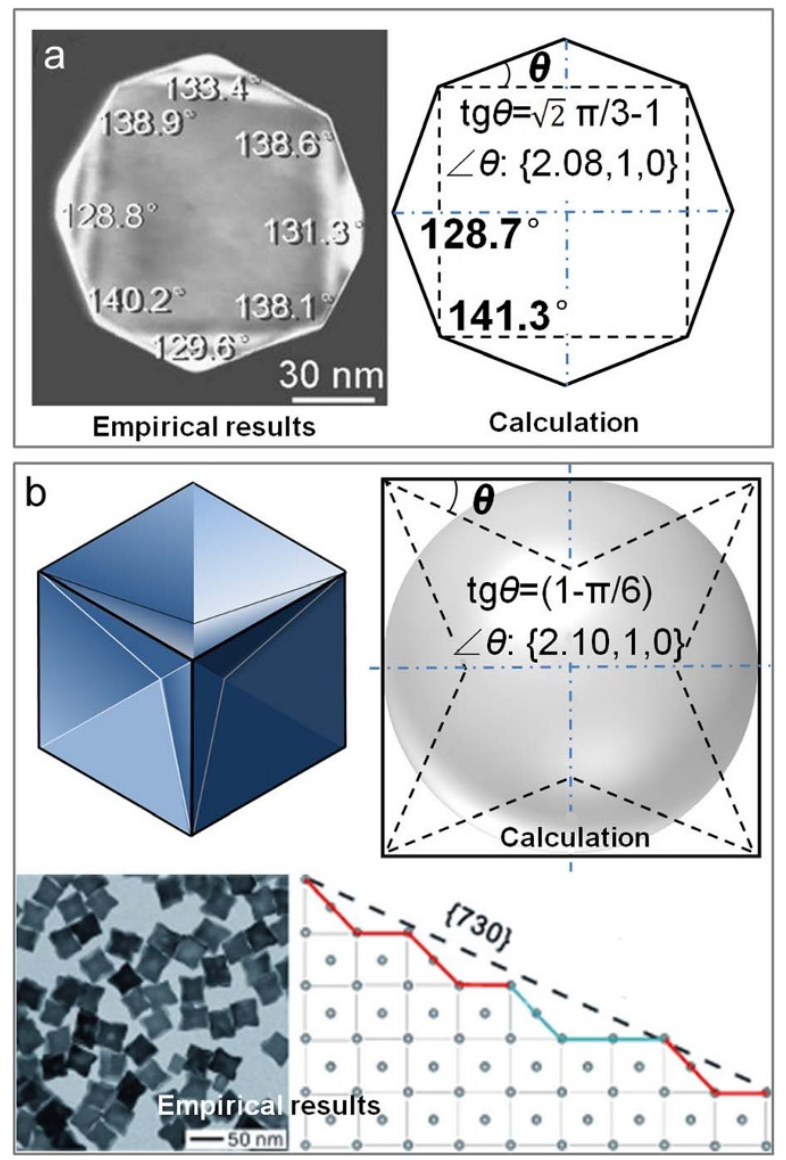

Figure $4 \mid$ Comparison between the calculations and the empirical results: convex tetrahexahedrons (a) and concave tetrahexahedrons (b) [the empirical result in Panel a is adapted from Ref. 8, Copyright 2007 of AIP; and the empirical result in Panel $\mathbf{b}$ is adapted from Ref. 1, Copyright 2011 of Wiley]. drons results from $\left\{1,\left(\frac{\sqrt{2}}{3} \pi-1\right), 0\right\}$, while that in concave tetrahexahedrons is from $\{1,(1-\pi / 6), 0\}$. Concerning the determinate term $\left(E_{s} \cdot S\right)$ for the concave tetrahexahedron, the surface area is $2.12 S_{0}$, which is relatively larger than that of the convex tetrahexahedron, while $E_{h k l}$ is almost equal to that of the convex tetrahedron. This observation suggests that the requirement of smaller surface areas is relatively peripheral here.

\section{Discussion}

The high-index surfaces can be expected during the transformation from continuous spheres to single crystal polyhedrons. The corresponding mechanism illuminates that the high-index facets may be obtained from a top-down method.

Nanostructures with continuous surfaces are common, such as nanorods of glasses, nano sphere-caps or nanodrops of liquids, and they can be crystallized into single crystals under certain conditions. Based on the above analysis, the transitions from an amorphous rod to a crystal rod with cubic structure and axial orientation can be designed, as demonstrated in Fig. 5. If the conditions are tuned to tighten the surface areas, the high-index surfaces of $\{1,(\pi / 2-1), 0\}$ may be expected. As shown in Fig. $5 \mathrm{a}$, the stable core of quadrangle can divide the exterior atoms/molecules into four topologically unconnected zones, and the exterior atoms/molecules will tune themselves via migrating and reach the equilibrium positions with equal surface energy and $D_{4}$ symmetry. The synthesized single crystal rod should be covered by the facets composed of (210) steps and (110) steps, and the index could be approximate to $\{740\}$. If the conditions tend to loosen the surface area, single crystal rods with the index of $\{1,(1-\pi / 4), 0\}$ are expected to form. If they are stable enough to avoid transformations to vicinal planes with lower surface energy, the presence of (410) steps and (510) steps can be expected (Fig. 5b). Similarly, more general transition processes involving other crystal structures and orientations can be also designed, as shown in Fig. 5c.

In summary, this work proposes a hypothesis to address the origin of the high-index surfaces in specific single nanocrystals, and suggests that the high-index surfaces are determined by the covariation of $E_{h k l}$ and $S$ in various systems. The geometrically determinate performances can be understood by migrating atoms/molecules on or
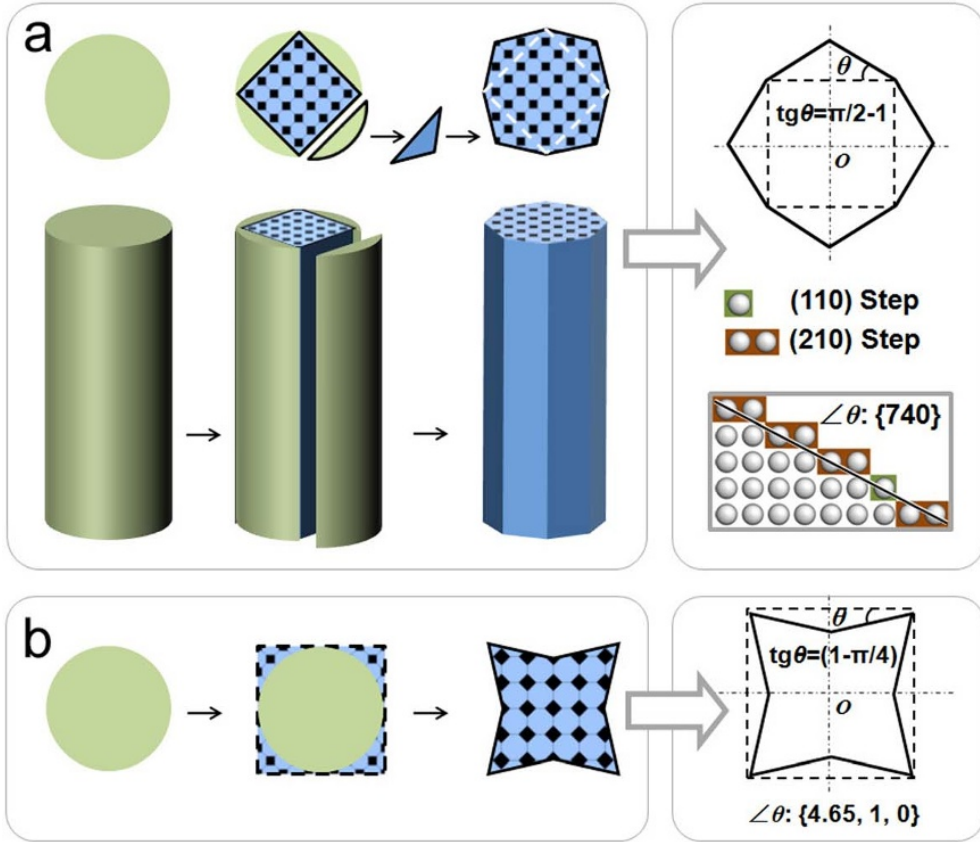

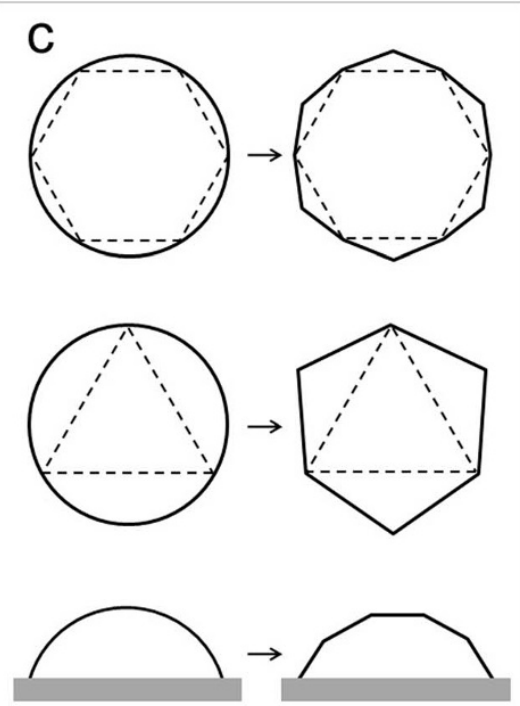

Amorphous
Crystal

Figure $5 \mid$ Designing the high-index surfaces: $\{1,(\pi / 2-1), 0\}(a),\{1,(1-\pi / 4), 0\}(b)$, and other potential approaches (c). 
near the continuous surfaces. The corresponding calculations are in good agreement with the empirical results. Our work highlights the crucial points to obtain the high-index surfaces in nanocrystals: (i) the structures with continuous surfaces are facilitative precursors for obtaining high-index surfaces, (ii) kinetic energy of the exterior atoms/molecules is required to be strong enough to overcome the binding energy and to move the exterior atoms/molecules in these cases, and (iii) the geometrically critical points control the highindex surfaces. From this hypothesis, a broad group of high-index surfaces in nanocrystals can be predicted. This present study would open up a novel way to guide the synthesis of catalyst with high-index surfaces at nanoscale.

1. Jin, M., Zhang, H., Xie, Z. \& Xia, Y. Palladium Concave Nanocubes with HighIndex Facets and Their Enhanced Catalytic Properties. Angew. Chem. Int. Ed. 50, 7850-7854 (2011).

2. Kang, Y. \& Murray, C. B. Synthesis and Electrocatalytic Properties of Cubic MnPt Nanocrystals (Nanocubes). J. Am. Chem. Soc. 132, 7568-7569 (2010).

3. Lu, C.-L., Prasad, K. S., Wu, H.-L., Ho, J.-A. A. \& Huang, M. H. Au nanocubedirected fabrication of $\mathrm{Au}-\mathrm{Pd}$ core - shell nanocrystals with tetrahexahedral, concave octahedral, and octahedral structures and their electrocatalytic activity. J. Am. Chem. Soc. 132, 14546-14553 (2010).

4. DeSantis, C. J., Peverly, A. A., Peters, D. G. \& Skrabalak, S. E. Octopods versus concave nanocrystals: Control of morphology by manipulating the kinetics of seeded growth via co-reduction. Nano Lett. 11, 2164-2168 (2011).

5. Tian, N., Zhou, Z.-Y. \& Sun, S.-G. Platinum metal catalysts of high-Index surfaces: from single-crystal planes to electrochemically shape-controlled nanoparticles. J. Phys. Chem. C 112,19801-19817 (2008).

6. Xia, Y., Xiong, Y., Lim, B. \& Skrabalak, S. Shape-controlled synthesis of metal nanocrystals: simple chemistry meets complex physics. Angew. Chem. Int. Ed. 48, 60-103 (2009).

7. Yu, T., Kim, Im, S. H. \& Park, O. Synthesis of Tetrahexahedral Gold Nanocrystals with High-Index Facets. Cryst. Growth Des. 10, 3321-3323 (2010).

8. Ding, Y. et al. Facets and surface relaxation of tetrahexahedral platinum nanocrystals. Appl. Phys. Lett. 91, 121901; DOI:http://dx.doi.org/10.1063/1. 2785953 (2007).

9. Ming, T. et al. Growth of tetrahexahedral gold nanocrystals with high-index facets. J. Am. Chem. Soc. 131, 16350-16351 (2009).

10. Tian, N., Zhou, Z.-Y., Sun, S.-G., Ding, Y. \& Wang, Z. L. Synthesis of tetrahexahedral platinum nanocrystals with high-index facets and high electrooxidation activity. Science 316, 732-735 (2007).

11. Tian, N., Zhou, Z.-Y., Yu, N.-F., Wang, L.-Y. \& Sun, S.-G. Direct electrodeposition of tetrahexahedral Pd nanocrystals with high-index facets and high catalytic activity for ethanol electrooxidation. J. Am. Chem. Soc. 132, 7580-7581 (2010).

12. Gontard, L. C. et al. Aberration-Corrected Imaging of Active Sites on Industrial Catalyst Nanoparticles. Angew. Chem. 119, 3757-3759 (2007).
13. Lee, S. W. et al. Role of surface steps of Pt nanoparticles on the electrochemical activity for oxygen reduction. J. Phys. Chem. Lett. 1, 1316-1320 (2010).

14. Zhou, Y. et al. Spontaneous symmetry breaking discovers the formation of aeroplane-like ZnO nanocrystals. Appl. Phys. Lett. 104, 121901-121903 (2014).

15. Zhou, Y., Zhang, J., Li, J. \& Zhang, B. Spontaneous symmetry breaking during formation of ZnO nanocrystals. J. Cryst. Growth 324, 225-228 (2011).

16. Huang, X., Tang, S., Zhang, H., Zhou, Z. \& Zheng, N. Controlled formation of concave tetrahedral/trigonal bipyramidal palladium nanocrystals. J. Am. Chem. Soc. 131, 13916-13917 (2009).

17. Huang, X., Zhao, Z., Fan, J., Tan, Y. \& Zheng, N. Amine-assisted synthesis of concave polyhedral platinum nanocrystals having $\{411\}$ high-index facets. J. Am Chem. Soc. 133, 4718-4721 (2011).

18. Yu, T., Kim, D. Y., Zhang, H. \& Xia, Y. Platinum Concave Nanocubes with High Index Facets and Their Enhanced Activity for Oxygen Reduction Reaction. Angew. Chem. Int. Ed. 50, 2773-2777 (2011).

19. Zhang, J. et al. Concave cubic gold nanocrystals with high-index facets. J. Am. Chem. Soc. 132, 14012-14014 (2010).

20. Zhang, J. et al. Synthesis of Concave Palladium Nanocubes with High-Index Surfaces and High Electrocatalytic Activities. Chem. -Eur. J. 17, 9915-9919 (2011)

\section{Acknowledgments}

The authors acknowledge the Natural Science Foundation of China (No. 51275508) and National Key Basic Research and Development (973) Program of China (No. 2012 CB932900 and No. 2013CB933401) for financial support.

\section{Author contributions}

Y.Z. conceived the idea, performed the calculations, wrote the paper and prepared the figures, J.Z., G.S. and J.L. discussed the work and reviewed the manuscript, and Y.Z. and G.S revised the manuscript.

\section{Additional information}

Competing financial interests: The authors declare no competing financial interests.

How to cite this article: Zhou, Y., Zhang, J., Su, G. \& Li, J. From sphere to polyhedron: A hypothesis on the formation of high-index surfaces in nanocrystals. Sci. Rep. 4, 6520; DOI:10.1038/srep06520 (2014).

This work is licensed under a Creative Commons Attribution-NonCommercialNoDerivs 4.0 International License. The images or other third party material in this article are included in the article's Creative Commons license, unless indicated otherwise in the credit line; if the material is not included under the Creative Commons license, users will need to obtain permission from the license holder in order to reproduce the material. To view a copy of this license, visit http:// creativecommons.org/licenses/by-nc-nd/4.0/ 